\section{Oliver Haas}

\section{Radiotherapy Treatment Planning: New System Approaches}

\author{
Advances in Industrial Control \\ Springer, Berlin 1998 \\ 220 pp.; USD 79.95 \\ ISBN 1-8523-3063-5
}

This book has grown out of the author's research on the optimization radiotherapy treatment planning using methods from control theory. It provides a clear, concise coverage of the aspects of radiotherapy treatment planning optimization from a control theory point of view. This work covers both deterministic and heuristic or guided random search techniques to solve a wide range of problems in radiation oncology physics, some of which include the beam orientation problem, the optimization of the best possible combination of beam weight and wedge angle, as well as the optimization of intensity-modulated treatment plans. The book is organized into seven chapters. After a brief introduction the author goes on to discuss beam characteristics and modeling considerations, the formulation of matrixbased beam models, solving the inverse problem in radiotherapy treatment planning, and hybrid genetic algorithms applied to radiotherapy treatment planning. The book ends with a very nice chapter on the experimental verification of the overall approach.

Since this book brings together topics from control engineering, computer science, and medical physics it is of interest to anyone working in these fields. However, the book is probably most useful to $\mathrm{PhD}$ students in medical physics that would like to gain a clear and thorough understanding of radiotherapy treatment plan optimization using control theory.

Wolfgang Tomé, Madison, Wisc.

\section{E.E. Volkes, H.M. Golumb}

\section{Oncologic Therapies}

Springer, Heidelberg 1999

$1236 \mathrm{pp}$.

ISBN 3-540-64052-5

This paperback is described as a handbook for physicians who treat cancer patients. It contains over 50 chapters that cover therapeutic modalities, complications, supportive care, quality of life, biostatistics, and more than 30 different cancer sites. The majority of the authors come from the University of Chicago. The material is mainly text with relatively few diagrams and illustrations. It is well referenced.

The chapters contain up-to-date and relevant information. Included are data on incidence, epidemiology, pathology, laboratory classification, and therapy for different stages and specific chemotherapy regimens. The chapter on non-Hodgkin's lymphoma goes into great detail on the various pathology classifications.

This compact handbook is very helpful to the house officer or trainee who wants to have a concise reference source on hand. The material is sufficient to provide a reasonable reference as to tests to order or define a tentative treatment plan.

Paul P. Carbone, Madison, Wisc.
John A. Hickman, Caroline Dive (eds)

\section{Apoptosis and Cancer Chemotherapy}

\author{
Humana Press, Totowa 1999 \\ 342 pp.; USD 99.50 \\ ISBN 0-896-03743-6
}

Over the past 2 decades more than 20 different molecules have been found to be involved in apoptosis, a mechanism of cell growth regulation that seems to be turned off in cancer cells. These molecules represent a broad spectrum of biologic factors including receptors, cytokine-mediated signals, and missing links to apoptosis. These targets are of potential value as sites for chemotherapeutic agents. This book provides 21 chapters by experts that try to put into perspective the real value of these findings. In general, they point out that the field is complex and that many of the experimental results are based on contrived experiments on cell lines that may have little to do with clonogenic survival of cancer cells of nonhematopoietic origin. The authors also point out that important mechanisms like p53 alterations may have tissue specificity that precludes a single mechanism of action. Moreover, they note that the exact mechanisms of DNA damage that trigger cell death are not clearly known. The authors represent many experts as well as young investigators in the field.

In the first chapter, J.M. Brown and B.G. Walters clearly state the issues, namely that short-term in vitro assays are dependent more on the rate of cell death rather than clonogenic survivals. In addition, they state that unlike the normal cells transformed by dominant oncogenes, many tumors already have selected cells for apoptotic resistant phenotypes. They go on to state that in real tumor cells 'p53 and bcl-2 play little or no role in the sensitivity of non-hematological cells to radiation or drugs'. These conclusions are based on clonogenic assays rather than cell death experiments. This theme is repeated by S.W. Lowe in the next chapter.

\section{KARGER}

(c) 1999 S. Karger AG, Basel

Fax + 41613061234

E-Mail karger@karger.ch www.karger.com
Accessible online at: www. karger.com/journals/ocl 
There are several detailed chapters looking at other mechanisms and their possible role in apoptosis, such as mismatch repair deficiency, c-abl tyrosine kinase, bcl-2, bax, bak, mitochondrial release of cytochrome c, CD95, IGF-IR, and microenvironment. There are also several chapters on specific cancers including prostate, ovarian, breast, neuroblastoma, and testicular cancer. The details are more speculative rather than factual as to how apoptosis plays a role in cell death.

Finally, Dr. J. Bertino, a clinician and pharmacologist, summarizes the problems of translating these laboratory results to the clinic. He points out that trying to restore normal p53 function by gene transfer is complicated because tumor cells are often disseminated and getting the normal genes to each cancer cell is daunting. He states that one needs to find the specific Achilles' heel for each tumor and target that mechanism. This task is extremely difficult since a single weakness is unlikely to be found for any cancer. More likely, a combination of approaches to each cancer will need to be done.

This book is of value to many scientists as a current reference to a complicated field. It also provides a balanced view of the field and warns investigators that complexity is the norm and over-simplification is a laboratory phenomenon.

Paul P. Carbone, Madison, Wisc.

W.N. Hait, D.A. August, B.G. Haffty (eds)

\section{Expert Consultations in Breast Cancer}

\author{
Critical Pathways and Clinical Decision Making \\ Dekker, New York 1999 \\ 512 pp.; USD 99.75 \\ ISBN 0-8247-1954-9
}

Breast cancer is a common cancer, affecting more than 180,000 women each year. Many advances have been made over the past 3 decades including mammography, breast conserving surgery, adjuvant chemotherapy, use of tamoxifen, genetic screening, and prevention strategies. Despite these advances, breast cancer presents in many different ways, and during its clinical course can affect many organ systems. Many issues remain to be resolved. The impact of age on treatment, role of immediate versus delayed reconstruction, management of ductal or lobular carcinoma in situ, hormonal replacement, and genetic testing are just some of the unresolved questions.

This book by Hait, August and Haffty is a gem for the individual oncologist or surgeon who comes across some of the less common clinical presentations. Using a case report type of presentation, a variety of experts discuss their approach to managing the unusual case. These presentations are short, but informative, with annotated references. Nineteen such case reports are included.

In addition, the book has six critical pathways for working up patients with lesions to diagnose noninvasive lesions, invasive cancer and metastatic disease. These pathways are presented simply in diagram form with specific recommendations.

An introductory section of the book has short chapters written by experts on the medical basis for decision making. There are concise reviews of particular topics such as pathology, diagnosis, staging, surgery, radiation, chemotherapy and genetics. These chapters are written in prose easily understood with relatively few tables and graphs. Key references are noted.
All in all this is a very valuable book for the practicing oncologist or the student of breast cancer. Because the material is concisely written, the important details are not buried deep in the text as in the large text books. This book should be written as a loose leaf edition, where chapters can be upgraded or rewritten as needed. Several parts are already dated, namely the use of high dose chemotherapy and stem cell replacement, as well as the results of the tamoxifen prevention trials.

Paul P. Carbone, Madison, Wisc.

\section{Al B. Benson (ed) \\ Gastrointestinal Oncology: Cancer Treatment and Research}

Kluwer, Boston/Dordrecht/London 1999

391 pp.; USD 365.00

ISBN 0-7923-8205-6

Gastrointestinal (GI) cancers represent $20 \%$ of all malignancies in the USA, affecting almost 250,000 people annually. They account for $25 \%$ of all cancer deaths. Surgery has been the mainstay of treatment for many years. Recently, chemotherapy and combined modality treatments with X-rays and drugs have been shown to improve the outcome. However, because GI cancers represent a spectrum of diseases ranging from the esophagus to the anus and including the liver and pancreas, the advances have come slowly and have been limited to a few sites. Another advance has come from the basic laboratories where the molecular basis of human carcinogenesis is being elucidated, defining individuals at increased risk.

This book is small in size and is not comprehensive; each site of disease is not detailed. Selected topics have been presented that focus on areas of recent advances in treatment or areas of research. The topics discussed are detailed with review of the literature. The references are extensive. Most chapters end with recommendations for therapy; however, those paragraphs are short and very nonspecific, making it hard for the oncologist to decide on specific approaches.

The choice of chapters and content appear to be odd. The first chapter deals with the management of esophageal cancer which is broad, encompassing etiology, epidemiology, staging, surgery, radiation, and chemotherapy. The chapter on gastric cancer focuses entirely on adjuvant therapy which has not been shown to be effective. The chapter on colorectal cancer centers on the drugs. The rectal cancer chapter is primarily about adjuvant therapy.

There is a long, detailed chapter on 'new trends in gastrointestinal surgical oncology' that is very complete. The chapter on the molecular basis of colorectal cancer focuses on therapy and overcoming drug resistance.

According to the editors, this book is aimed at the multidisciplinary team managing patients with GI cancer, including medical specialists, nurses, and trainees. Unfortunately, the book is not complete, nor is it written in an explanatory mode. It spends a lot of time summarizing old trials, most of which were negative. The chapters are not well integrated. The material presented and the subsection titles are not uniform. If this were part of a series which plans to explore other aspects of GI cancers, it would be important. Standing alone it has less of an impact.

Paul P. Carbone, Madison, Wisc. 